\title{
La recepción de Voces usadas en Chile (1900) de Aníbal Echeverría y Reyes entre sus contemporáneos*
}

\author{
Dario Rojas Gallardo y Tania Avilés Vergara** \\ Universidad de Chile
}

\begin{abstract}
Resumen
En el presente artículo analizamos cómo fue valorado entre sus contemporáneos Voces usadas en Chile (1900), del abogado y lingüista aficionado chileno Aníbal Echeverría y Reyes, según muestra un corpus de seis textos críticos de esta obra fechados entre 1899 y 1905 . Algunos de estos textos fueron publicados en la prensa chilena y estadounidense, y el resto en forma de libro. Entre sus autores se hallan Rodolfo Lenz, Miguel Luis Amunátegui Reyes y Fidelis del Solar, quienes desempeñaron papeles importantes en el desarrollo de la lingüística en Chile. Concluimos que la recepción de la obra de Echeverría fue mayormente negativa porque los comentaristas la interpretaron como una obra prescriptiva que adolecía de defectos fundamentales para cumplir con su propósito
\end{abstract}

* Financiado por Programa U-APOYA Línea 2: Concurso de Proyectos de Investigación en Ciencias Sociales, Humanidades, Artes y Educación VID 2011, código SOC U-SOC-11/12 de la Universidad de Chile.

** Para correspondencia dirigirse a: Darío Rojas Gallardo (darioroj@u.uchile.cl) o Tania Avilés Vergara (taviles@ug.uchile.cl), Departamento de Lingüística, Facultad de Filosofía y Humanidades, Universidad de Chile, Ignacio Carrera Pinto 1025, Nuñoa, Santiago, Chile. Tania Avilés Vergara es Becaria de la Comisión Nacional de Investigación Científica y Tecnológica de Chile (CONICYT). 
normativo, interpretación congruente con el periodo de la lexicografía y la lingüística chilena en que se enmarca, llamado precientífico por Matus (1994) y caracterizado por un marcado interés normativista. Entre las críticas más abundantes sobresale el desacuerdo sobre el tratamiento normativo dado a ítems particulares, lo cual interpretamos en términos de una negociación de normas connatural a una comunidad lingüística que atraviesa un proceso de estandarización, como era el Chile hispanohablante de fines del siglo XIX.

Palabras clave: Voces usadas en Chile, Aníbal Echeverría y Reyes, historiografía lingüística, ideologías lingüísticas, metalexicografía, diccionarios de provincialismos.

\title{
The RECEPTION of ANíbal ECHEVERRÍA y Reyes's Voces USADAS EN CHILE (1900) BY HIS CONTEMPORARIES
}

\begin{abstract}
This paper analyzes how Voces usadas en Chile, a lexicographical work written by Chilean lawyer and amateur linguist Aníbal Echeverría y Reyes and published in 1900, was received by his contemporaries, as it can be seen in a corpus of six critical texts dated between 1899 and 1905. Some of these critical texts were published in Chilean and American press and the rest in book form. Among the authors of these texts are Rodolfo Lenz, Miguel Luis Amunátegui Reyes and Fidelis del Solar, all of which played a significant role in the development of linguistics in Chile. We conclude that the reception of Echeverría's work was mainly negative because most commentators interpreted it as a prescriptive work whose normative function was impeded by fundamental faults. This interpretation can be explained in the context of the period of Chilean lexicography and linguistics to which these texts belong, the pre-scientific period, as Matus (1994) calls it, characterized by a strong normative interest. Among the most frequent criticisms, disagreements concerning the normative treatment of specific items stand out. We interpret this as a case of norm negotiation, which we deem inherent in a linguistic community undergoing a standardization process, as it was the case of late 19th century's Spanish-speaking Chile.
\end{abstract}

Key words: Voces usadas en Chile; Aníbal Echeverría y Reyes, linguistic historiography, language ideologies, metalexicography, dictionaries of provincialisms.

Recibido: 20/08/2012 Aceptado: 28/11/2012 


\section{INTRODUCCIÓN}

En el presente estudio analizamos cómo fue valorado Voces usadas en Chile (1900), del abogado Aníbal Echeverría y Reyes, por parte de sus contemporáneos chilenos y extranjeros. Nuestro análisis se basa en un corpus de textos críticos de dicha obra, que se encuentran fechados entre 1899 y 1905.

Tanto la obra objeto de comentario como los comentaristas quedan enmarcados en el periodo de la lexicografía chilena llamado precientífico por Matus (1994) ${ }^{1}$. Este periodo, según dicho autor, se caracteriza por la falta de rigurosidad metodológica, por la autoría de aficionados y no de profesionales del estudio del lenguaje, $\mathrm{y}$, principalmente, por el propósito normativo que guiaba a estas obras, como se puede apreciar en Rodríguez (1979 [1875]) y Ortúzar (1893). El primero de estos autores, en el "Prólogo" de su obra, apunta que "la Gramática castellana [...] no es mas que el arte de hablar y escribir correctamente el español" (Rodríguez 1979 [1875]: viii) y que el propósito de su obra es brindar a los estudiosos "un fácil medio de evitar los errores mas comunes que, hablando o escribiendo, se cometen en nuestro pais en materia de lenguaje" (Rodríguez 1979 [1875]: viii). El sacerdote salesiano, por su parte, señala que su trabajo se origina en la necesidad de contar con "un pequeño vocabulario, donde [...] puedan consultarse las más importantes correcciones de lenguaje, conforme á la enseñanza de renombrados hablistas" (Ortúzar 1893: xi). Varios años más tarde, Rodolfo Lenz (1926: 9) recuerda la extrañeza que le causó, al llegar a Chile en 1890, que en su comunidad adoptiva los diccionarios se usasen no simplemente para conocer el significado de alguna palabra, como se hacía en la Alemania de la época, sino para conocer la ortografía o, la mayoría de las veces, determinar el carácter "correcto" o "incorrecto" de una palabra. Estas y otras afirmaciones y anécdotas dan cuenta de la perspectiva normativista que guiaba los estudios del lenguaje y la reflexión metalingüística en el Chile de fines del siglo XIX no solo en su vertiente diccionarística, sino también en otras disciplinas lingüísticas.

La perspectiva normativista respondía al clima intelectual suscitado por los movimientos independentistas americanos y los problemas que estos procesos planteaban en diversos ámbitos culturales, entre ellos el lingüístico.

1 En nuestro análisis hemos prescindido de comentarios posteriores como los de Medina (1928: 10-11) y Rojas Carrasco (1940: 94-95), que pueden considerarse más bien propios de la etapa de transición hacia la lexicografía moderna, según esta misma periodización. 
Andrés Bello, figura gravitante no solo en la conformación de las ideas sobre el lenguaje en Chile sino también influyente en muchos otros ámbitos de la vida cultural, afirmó famosamente:

Juzgo importante la conservación de la lengua de nuestros padres en su posible pureza, como un medio providencial de comunicación y un vínculo de fraternidad entre las varias naciones de origen español derramadas sobre los dos continentes. (Bello 1847: x-xi)

La preocupación por la unidad de la lengua, reflejada en la cita anterior, conduce fácilmente a una preocupación por la corrección idiomática: la lengua se transforma en un ideal platónico (el español correcto, ejemplar, castizo) sobre cuyo trasfondo son evaluadas las conductas idiomáticas de los individuos de una comunidad. Las únicas formas lingüísticas que adquieren legitimidad, en este trasfondo, son las "correctas", es decir, las que se ajustan al ideal de lengua, y se entiende que la unidad de la lengua se conservará solo en la medida en que la conducta idiomática de los miembros de la comunidad tienda hacia este ideal.

En este contexto o clima de opinión lingüística de tipo evaluativo, Voces usadas en Chile sobresale por su aparente objetividad en el tratamiento de las unidades léxicas que recoge. Esto la ha hecho ser considerada como una obra sui géneris, pues, en apariencia, carece de los acentuados tintes normativistas de obras como las mencionadas. Sin embargo, aparte de los comentarios de Solar (1900) y del informe de Lenz y Diez al Consejo de Instrucción Pública (considerados en sus estudios por Becerra, Castro y Garrido 2007 y Chávez 2009), no se han analizado otras obras críticas de Voces usadas en Chile, por lo cual aún no sabemos a ciencia cierta cómo fue recibida esta obra por parte de sus contemporáneos. Nuestro estudio considera un repertorio de críticas más amplio que los utilizados antes: el informe de Lenz y Diez, fechado en 1899 y reproducido en Echeverría (1900: vii-xi), Solar (1900), Mario (1900), Anónimo (1900), Amunátegui Reyes (1902) y Lenz (1987 [1905-1910]). Entre sus autores se hallan algunos estudiosos que desempeñaron papeles importantes en el desarrollo de la lingüística en Chile.

Consideramos que estudiar cómo sus contemporáneos valoraron la obra de Echeverría, y sobre la base de qué ideas hicieron sus juicios, contribuye a revelar cuál era la idea o imagen del diccionario que existía en el Chile de fines del siglo XIX y comienzos del Xx. Nuestro estudio, entonces, se enmarca en dos líneas de investigación. Primero, tiene como marco la metalexicografia o estudio de los diccionarios desde un punto de vista descriptivo o teórico (Azorín Fernández 2003: 38-44). De entre las áreas de estudio general que, según Wiegand (1984), componen la metalexicografía, nuestra investigación concierne a la historia de la lexicografía y a la crítica 
de diccionarios; específicamente, en cuanto a esta última área, se trataría de una parte de la historia de la crítica de diccionarios, o, puesto en otras palabras, de la historia de la metalexicografia (Hausmann 1989). En segundo lugar, nuestra investigación concierne igualmente a la historiografía de las ideologías lingüísticas (Blommaert 1999: 1; Kroskrity 2010: 201-203), que se ocupa del estudio de cómo se conforman, reproducen y transforman a lo largo del tiempo estos conjuntos de creencias acerca del lenguaje, considerando su contexto histórico, político y social (Blommaert 1999: 2; Coupland \& Jaworski, 2004: 37). Los diccionarios suelen ser considerados por los no especialistas como representantes del lenguaje en su totalidad, y, más aún, como contenedores del lenguaje "verdadero" o legítimo (Béjoint 2000: 116-121; Lara 1997: 64-66), de modo que la reflexión sobre los diccionarios conlleva, de una u otra manera, ideas sobre el lenguaje. Es interesante, sobre todo, considerar las ideologías lingüísticas que sostuvieron especialistas o semiespecialistas de épocas pasadas, pues se ha encontrado que las ideas que los legos tienen en la actualidad acerca del lenguaje tienen huellas de las sostenidas por los intelectuales de generaciones anteriores y que han quedado obsoletas desde el punto de vista científico (Wilton y Wochele 2011).

En las secciones siguientes, presentaremos el análisis según el orden cronológico de los textos, para cada autor por separado, y luego ofreceremos una clasificación y síntesis de las principales ideas identificadas.

\section{EL INFORME DE LENZ Y DIEZ AL CONSEJO DE INSTRUCCIÓN PÚBLICA (1899)}

En el año 1900, Aníbal Echeverría y Reyes, luego de un extenso proceso de planificación y revisión, consiguió publicar Voces usadas en Chile como anexo de los Anales de la Universidad de Chile. Para obtener este beneficio, Echeverría debió solicitarlo formalmente al Consejo de Instrucción Pública. Este organismo delegó en Rodolfo Lenz y Antonio Diez, miembros de la Facultad de Filosofía, Humanidades y Bellas Artes, la elaboración de un informe respecto de los méritos del trabajo de Echeverría. Desde un primer momento, de hecho, Echeverría había comprometido la ayuda de Lenz, como se puede ver en las cartas que el abogado le envió al filólogo alemán durante el proceso preparatorio de la publicación (Rojas 2011). Este informe fue incluido como parte de la edición final de Voces usadas en Chile, en sus primeras páginas. 
Se trata de la primera reacción valorativa pública a la aparición del trabajo de Echeverría. La valoración, como puede suponerse, es positiva en lo general, pues el Consejo de Instrucción Pública admitió la publicación de la obra a expensas de fondos estatales:

... ahora presenta su libro en forma tal, que bien puede considerársele como el mejor de cuantos sobre la materia se han publicado en el pais. [...] La prolijidad del trabajo honra por igual a la paciencia i laboriosidad del autor. Las omisiones i deficiencias que en esta obra tan interesante hemos notado, no son de tal naturaleza que puedan afectar a la obra en conjunto; ellas son inevitables en trabajos de esta índole i pueden fácilmente subsanarse. (Echeverría 1900: IX-XI)

En lo particular, Lenz y Diez comentan favorablemente la inclusión de una bibliografía que consideran "un verdadero modelo en el género" (Echeverría 1900: IX). Señalan que los primeros capítulos, relativos a características fónicas y gramaticales del español de Chile, podrían haber sido dispuestas de un modo distinto, según exigiría "el riguroso método científico de la filolojía moderna" (Echeverría 1900: x), pero conceden que la presentación es clara y que se puede aceptar por ser el propósito del autor principalmente práctico y desear llegar al público general.

El informe no entra en consideraciones particulares respecto del contenido. Lenz, sin embargo, algunos años más tarde (véase la sección 7 del presente artículo) criticará especialmente la sección lexicológica. Puede pensarse que algunas de estas observaciones ya habían sido planteadas por Lenz al autor antes de publicarse la obra, como se desprende de lo dicho en el mismo informe así como en la correspondencia entre Echeverría y el filólogo alemán (Rojas 2011).

\section{LA CRÍTICA DE FIDELIS DEL SOLAR (1900)}

Fidelis del Solar, comentarista también del Diccionario de chilenismos de Zorobabel Rodríguez (Solar 1876) y autor de otros opúsculos ortográficos y lexicológicos, comienza alabando a Echeverría por haber emprendido una obra que tiene valor cívico, pues "cumple con la noble misión de sacar a sus compatriotas del estado de postración en que se encuentran: les pone delante las incorrecciones de lenguaje en que incurre el pueblo con el laudable propósito de educarle e instruirle" (Solar 1900: 4). Como puede 
apreciarse, el propósito normativo y pedagógico de Voces usadas en Chile, compartido por la mayor parte de los diccionarios y gramáticas publicados en Chile en la época (Rojas 2010), es considerado por Solar como su cualidad más valorable.

En relación con el propósito pedagógico de exhibir y denunciar usos considerados incorrectos, para Solar resulta loable, asimismo, la cantidad y diversidad de voces incluidas: "Considero la obra que analizo como lo más completo en la materia que se ha publicado en Chile" (Solar 1900: 7). Este autor hace hincapié en que el repertorio reunido por Echeverría supera ampliamente a Rodríguez (1875): mientras que el primero recoge más de 4000 voces, el segundo supera por poco el millar. En cuanto a la diversidad, valora positivamente que Voces usadas en Chile incluya no solo chilenismos, sino también americanismos, neologismos y extranjerismos. En estos comentarios, Solar hace una interpretación explícita de las intenciones del autor, influenciada, no obstante, por su opinión de que muchas de estas voces pueden incorporarse al Diccionario académico:

... chilenismos, voces peculiares del habla chilena, sean o no dignas de figurar en el Diccionario oficial: el autor las exhibe; todas las que ha podido obtener están allí para que juzgue el consultor por sí mismo las aceptables y rechace las demás del lenguaje por absurdas e inútiles. (Solar 1900: 5)

Asimismo este autor, tal como habían hecho Lenz y Diez en su informe, considera muy útil la inclusión de la bibliografía de obras de referencia.

Solar se ocupa también de algunas cuestiones relativas a la técnica lexicográfica empleada en Voces usadas en Chile. En primer lugar, considera que las definiciones son poco claras debido a su concisión ("bien valdría la pena de haber esplanado un poco más las ideas que esos artículos contienen"; Solar 1900: 7), y correlaciona este rasgo con la inclusión de un gran caudal de voces en poco espacio. En las definiciones de los extranjerismos y galicismos, echa de menos la entrega de un equivalente que se pueda usar en lugar de ellos. Echeverría en estos casos define con frecuencia en forma perifrástica ${ }^{2}$, pero Solar considera que, para el propósito pedagógico y normativo de la obra, hubiera sido más adecuado dar un término castellano equivalente. De

\footnotetext{
2 Por ejemplo, en back ("Uno de los dos jugadores en el Football, que están delante del goal-keeper, para defender su lado") y chantage ("Esplotacion monetaria de secretos ajenos, que consiste en amenazar con la publicacion de actos íntimos, si no se paga cierta suma de dinero").
} 
no darse un equivalente hispánico, "esto hace que el consultor se encariñe con el estranjerismo en vez de repulsarlo" (Solar 1900: 8). Los únicos casos en que cree aceptable definir extranjerismos en forma perifrástica son aquellos en que no se puede encontrar un equivalente, como el galicismo chantage.

En segundo lugar, hace la observación de que las abreviaturas empleadas en el apartado lexicológico son a veces ambiguas y poco claras. Propone una serie de modificaciones, "conforme con la práctica más jeneral en los diccionarios" (Solar 1900: 10), tales como diferenciar tipográficamente las clases de marcas (cursivas para las marcas gramaticales: adj., $a d v$., etc., $\mathrm{y}$ versalitas para las marcas de valoración normativa: AM., JERM., etc.) y hacer más extensas algunas para que sean más transparentes ( $a$. > ANT. 'anticuado', ch. > CHIL. 'chilenismo', etc.).

La mayor parte del opúsculo de Solar está dedicada a la crítica del tratamiento particular de ítems léxicos. Primero hace acotaciones sobre chilenismos falsos, que "han sido tomadas por chilenismos, siendo en realidad muy castizas" (Solar 1900: 11). Entre estos se encuentran abastero, cobrar, ama seca, pollera, rocambor, arbolito, agua de lavanda, celemín, bodegón y otras que en total suman 21 voces. Para fundamentar sus observaciones, se basa bien en diccionarios españoles o en citas de autores peninsulares tales como Cervantes, Pardo Bazán, Fernán Caballero, Pérez Galdós y Bretón de los Herreros.

Luego señala un grupo de supresiones necesarias, "palabras que [...], a mi juicio, no deben figurar en él” (Solar 1900: 29), especialmente algunos neologismos que ya aparecen en los diccionarios o que no vale la pena incluir. Por ejemplo, para Solar no se justifica la inclusión de los nombres de vinos chilenos (Urmeneta, Subercaseaux, etc.), ni considerar como barbarismos agua de Colonia, agua Florida y agua blanca, cuyos referentes han pasado a ser comunes, además de basarse en recursos metonímicos muy frecuentes en la lengua española. Por otro lado, Solar no está de acuerdo con la solicitud de incluir en el Diccionario académico algunos neologismos tales como afeitarse, pues este ya está, o hacer bombo, que ya aparecía registrado en otros diccionarios hispánicos. En este caso, culpa del error de Echeverría a la atribución de un significado muy limitado, lo que se refleja en la concisión de las definiciones ${ }^{3}$.

\footnotetext{
3 En relación con esta crítica, Solar compara las definiciones de (hacer) bombo que se encuentran en Echeverría: "Bambolla, escesiva ostentacion" y en el diccionario de Zerolo: "Elojio desmesurado i jeneralmente inmerecido que se hace de algún individuo o de sus obras". Cierra su comentario con un lamento: "iSiempre la demasiada concision en su obra!" (Solar 1900: 31).
} 
En esta sección reacciona airadamente contra lo que considera un liberalismo excesivo por parte del autor, solicitando la supresión de las voces vulgares, que prefiere no nombrar sino localizarlas mediante número de página, columna y artículo:

Son espresiones tan soeces, por mas chilenas que sean las más, que no me atrevería a nombrarlas por decencia. Francamente, no pensé jamás que ningún vocabulario formase caudal de ellas. Pertenecen al lenguaje de la hez del pueblo y figuran algunas archi-españolas que, si bien son muy frecuentes en España y sus colonias, ningún lexicógrafo las ha incluido en su diccionario. (Solar 1900: 32)

No es bastante esplicación la que da el autor en su prólogo para dar cabida a tan repugnantes espresiones, que, como he dicho antes, nadie hasta ahora ha tenido cara para presentarlas en obras serias; que si bien se toleran muchas en obras de esta naturaleza que pertenecen al género pornográfico, ninguna de las que condeno se halla en este caso. (Solar 1900: 33)

Las expresiones a las que se refiere Solar son algunas que Echeverría no considera vulgarismos, como carajo (interjección equivalente de 'caramba') y condom ('preservativo'), y otras que el autor del repertorio marca explícitamente como voces vulgares: culear, chincol, chucha, chulloca, huevada, huevón, huevos, joder, ña mica, pájaro, pico, picha y pichula.

Por último, propone agregaciones y rectificaciones. En esta sección añade algunos barbarismos (peñazcazo, noscada (nuez), contagioso, garuga, presupuestar), chilenismos (trompa, golondrina, gasfiter, gasfitero, gasfitería, quiltrín, quirinca, sango, cocho, ulpo, chercán, judas), extranjerismos innecesarios (fourriere) y necesarios (morgue, así como el ya dicho gasfiter y sus derivados), corrige (capitoso no es chilenismo, esprit y no sprit, starter y no stater) y agrega acepciones (paguacha).

\section{LOS REPAROS DE MARIO (1900)}

Entre los números 8122 y 8139 del periódico santiaguino El Porvenir apareció una serie de artículos titulados "Reparos a Voces usadas en Chile por Aníbal Echeverría y Reyes", firmados por Mario. Según Amunátegui Reyes (1902), Mario es seudónimo empleado por el presbítero José Ramón Saavedra, autor de algunas obras de gramática (Rojas Carrasco 1940: 216-220). En 
estos artículos, Mario se propone comentar la sección "Voces" de la obra de Echeverría. La publicación de estos reparos suscita la respuesta de un autor que firma con el seudónimo Lucio Flavio, que se muestra completamente de acuerdo con Mario, excepto en el tratamiento de la voz minorista. La mayor parte de los artículos de Mario están dedicados a comentar en particular la definición o el estatus normativo de alguna de las voces recogidas por Echeverría. Antes de presentar estas críticas, el autor hace dos observaciones generales. Primero, un asunto microestructural: la adición de la abreviatura $e$ (de extranjerismo) en los artículos correspondientes a extranjerismos le parece redundante, pues su carácter extranjero ya queda señalado por otras abreviaturas (como ang). En la segunda observación general denuncia que "casi todas, ó por lo menos la mayor parte de tales voces ó locuciones del Vocabulario, no son chilenismos, es decir, palabras ó modismos usados en Chile por los chilenos cuando se expresan en castellano". Con esto apunta a que dichos términos extranjeros en su opinión no tenían uso generalizado en el español de Chile de la época. Más adelante señala que, si se encuentra equivocado y dicha cantidad de extranjerismos realmente tienen vigencia de uso, entonces no cabe más que lamentarse por el futuro del idioma español. Para ilustrar su afirmación, reproduce una lista de 157 anglicismos recogidos por Echeverría. Luego se dedica a comentar una docena de estas voces. Por ejemplo, señala que algunos de estos anglicismos ya se encuentran registrados en el Diccionario académico (biftec, rosbif, esplín, tranvía, vagón y yate), por lo cual "forman ya el tesoro de la lengua, y pueden usarse dondequiera se hable castellano". Otros términos han sido definidos de manera equivocada (beafsteak, box, dreack, express). También observa a veces poco esmero en el lenguaje usado por el propio Echeverría para definir. Al finalizar la primera entrega, acusa a Lenz y Diez de poco prolijos en la revisión de la obra.

En la segunda entrega (número 8123) reproduce una lista de poco más de 300 galicismos contenidos en Voces usadas en Chile. Hace dos comentarios generales. Primero, que quienes usan estos términos extranjeros "merecen que, en todo país donde se hable castellano, sean excluidas del trato social como furibundos galiparlistas y perturbadores del idioma nacional". Segundo, que hay 34 voces de esa lista que, por encontrarse en el Diccionario de la RAE, "dejan de ser galicismos", y que hay otras tantas expresiones que Mario sospecha no lo son realmente. Finalmente, critica el tratamiento que Echeverría da a cinco galicismos. En el número 8124 presenta dos breves listas de germanismos (14) e italianismos (29). Sobre los primeros solo critica la precisión de los equivalentes que Echeverría da para Reichstag (Congreso y Cámara). En cuanto a los italianismos, cuestiona el carácter extranjero de estafermo (que "es ya castellano"). 
A partir de la edición 8126 de El Porvenir el interés de Mario se traslada hacia las voces de origen español, con el propósito de "ver si este opúsculo merece ser censurado con demasiada justicia". Entre los números 8126 y 8129,8131 y 8134 y concluyendo en el número 8139 , el autor comenta críticamente 214 voces de este tipo. En la última de estas ediciones hace una valoración global de la obra. Señala, en primer lugar, que probablemente los puntos cuestionables sean muchos más, e invita a que personas más instruidas o con mejor ojo hagan su propia revisión. Además hace una comparación explícita entre la obra de Echeverría y los diccionarios de Rodríguez y Ortúzar. Declara que, aunque supere cuantitativamente a sus predecesores,

se les queda muy atrás en filología, en gramática y en lógica. Aquellos Diccionarios, ó hacen indagaciones sobre la etimología de los chilenismos, si vienen del araucano, del quichua o del aimará, ó dan reglas para el uso de esas voces en el idioma, ó en sus definiciones se ajustan a la más precisa pauta filosófica. El Glosario, al revés, no toma en consideración el origen de esas palabras, desconoce a veces su existencia en el idioma, $\mathrm{y}$, lo que es peor, las define muy de continuo con una inexactitud que da pena. (El Porvenir 8139: 1)

Aquí volvemos a encontrar una de las críticas más recurrentes a Voces usadas en Chile: la imprecisión o la brevedad de sus definiciones.

Al igual que la mayoría de los comentaristas de Echeverría, Mario también dedica un párrafo a reprocharle el haber incluido voces vulgares:

Parece que el autor tuviese a gala en levantar del fango del lenguaje popular ciertas voces que designan objetos ó actos que no pueden nombrarse sin rubor, como si creyese realzar el mérito de la obra con la abundancia de tales palabras [...]. Pues bien, al manifestar el Glosario todas esas voces, ha traicionado la timidez de las personas pudorosas y dado alas a la desvergüenza de los disolutos. Y no hay que escudarse con el deber del lexicógrafo. Si una triste necesidad lo obliga a sentar ciertos vocablos, no la hay para seguir al lenguaje por los matices y ondulaciones por donde lo lleven vagarosas fantasías ó destemplados arranques del desenfreno. (El Porvenir 8139: 1)

En la conclusión reitera de forma aún más acusada la crítica a Lenz y Diez (especialmente al primero de ellos) que había venido asomando en entregas anteriores. Comenta sarcásticamente el que estos filólogos hayan apreciado con muchos elogios la obra de Echeverría en su informe al Consejo de Instrucción Pública, acusa a Lenz de ignorancia del idioma que enseña, y le atribuye desprolijidad por haber pasado por alto tantos errores notados por 
el propio Mario, asunto más grave aún si se considera que Echeverría había dedicado su obra a la RAE y que pronto la leerían los académicos españoles.

Sobre este último asunto en particular, en el número 8131 un lector de El Porvenir, que firma como Lucio Flavio, remite a Mario una breve carta en que le ruega encarecidamente que envíe a la RAE copias de sus reparos, con el fin de subsanar el daño infligido por Echeverría a la imagen de la lingüística chilena antes los ojos de los españoles. En el número 8135 Mario se niega humildemente señalando que "lejos de pensar yo que esos articulejos [sus propios reparos] puedan suministrar la más pequeña luz á la Real Academia, juzgo, al contrario, que de ésta debieran aquéllos recibirla". En la edición 8136 Lucio Flavio insiste: "¿Cómo habría Ud. de dejar ahora que al postrero de esos trabajos que de Chile han partido y con tanta osadía no le siguiesen los merecidos Reparos que Ud. le ha hecho? Este punto es de puro amor nacional".

En síntesis, Mario hace una de las críticas más detalladas y severas de la obra de Echeverría.

\section{LA RESEÑA ANÓNIMA DE THE NATION (1900)}

El semanario norteamericano The Nation publicó, el mismo año de aparición de la obra, una breve reseña de Voces usadas en Chile (Anónimo 1900).

Esta reseña considera que el repertorio de Echeverría es digno de atención por tratarse de una muestra del "triunfo pacífico de la lengua inglesa en lugares tan distantes de su solar originario" (traducción nuestra). Observa influencia no solo del inglés, sino también de otras lenguas extranjeras, tales como el francés (buqué, menu), el italiano (dilettante, fanciulla) y el alemán (bock, kirsch): no obstante, estas lenguas no logran superar la influencia ejercida por la primera en el español de Chile.

Los anglicismos registrados en Voces usadas en Chile, en opinión del autor de la reseña, son reveladores de la influencia cultural anglosajona: "Listar cabalmente las palabras del inglés adoptadas en el habla local y general de Chile y sus implicancias sociológicas y sicológicas, sería, en cierto sentido, resumir la historia de nuestra raza" (traducción nuestra). Por ejemplo, voces del ámbito deportivo (sport, foot-ball, cricket, box) y algunos deportes que se encuentran particularmente bien representados (el fútbol y la equitación); la sociedad (club, flirt, high-life, dandy); la política (bill, budget, self-government); la gastronomía, en especial la etílica (bar, coctail, punch); el transporte y el comercio (dock, check, stok, wagon, tranway); y muchas otras de difusión mundial (beefteck, clown, dollar, gentleman, snob); 
todas ellas revelan ámbitos culturales en los cuales los anglosajones habían ejercido una fuerte influencia en el resto del mundo. El autor de la reseña atribuye en particular al inglés norteamericano la presencia de Lager beer, pilsener, coctail, lynchar, yankee y otros.

Para el reseñador, la inclusión de algunos extranjerismos resulta reveladora del carácter actualizado de Voces usadas en Chile. Por ejemplo, uitlanders ('Estranjeros radicados en el Transvaal') y boers ('Campesinos de orijen holandés, que han constituido la República del Transvaal, en el Sur del África') aluden a una realidad relativamente novedosa en el momento de publicación de la obra: las minas de oro del Transvaal habían sido descubiertas en 1886, y en el 1900 la segunda guerra de los Bóers se hallaba en pleno desarrollo.

El resto de los comentarios alude a los préstamos provenientes de lenguas indígenas americanas y, por último, a los vulgarismos que se listan en los primeros capítulos (dulce $>$ urse, podrido $>$ poirio). Asimismo, valora en general la información contenida en los capítulos relativos a cuestiones fónicas y gramaticales. También menciona que la amplitud de la bibliografía consignada por Echeverría muestra la dedicación que puso en su trabajo. Se hace eco, igualmente, de la opinión expresada por Lenz y Diez en su informe, declarando que Voces usadas en Chile es "el mejor [libro de su clase] publicado en Chile hasta ahora" (traducción nuestra).

\section{AMUNÁTEGUI REYES, CRÍTICAS Y CHARLAS (1902)}

Dentro de toda su producción intelectual, las obras lingüísticas de Miguel Luis Amunátegui Reyes destacan por su abundancia y por la diversidad de intereses que revelan: gramática, ortografía, léxico y enseñanza de la lengua materna (Silva Castro 1951: 59-133). Además de su crítica a Voces usadas en Chile, publicó varios años más tarde un extenso comentario del diccionario de Ortúzar (Amunátegui Reyes 1924-1927).

La primera característica de Voces usadas en Chile que merece un fuerte reparo por parte de Amunátegui Reyes es la inclusión de voces tabuizadas:

En un Prólogo destinado a manifestar el plan de la obra, el autor se apresura a dar una explicacion por haber intercalado en el glosario un buen número de voces torpes i groseras. Estoi mui distante de pensar que un diccionario deba ser pudibundo hasta el estremo de omitir todos aquellos vocablos que denoten ideas poco decentes. Este pudor exajerado nos impediría conocer el verdadero sentido de ciertas dicciones de esta 
especie, tomadas ordinariamente del lenguaje técnico i vulgarizadas por necesidad. Pero de ahí a recoger todos aquellos términos que nacen i viven principalmente en el lupanar i en la taberna i que solo asoman en labios soeces, hai una distancia enorme. Jamas tales palabras han merecido el honor de ser estampadas en letras de molde, i por lo tanto no debemos empeñarnos en que salgan de esa atmósfera oscura i viciada que las ha enjendrado. Por otra parte, las mas de estas perniciosas lucubraciones no son otra cosa que grotescas metáforas que no habría razon para considerar como voces especiales. (Amunátegui Reyes 1902: 118-119)

Luego, Amunátegui Reyes dedica varias páginas a criticar el contenido de los capítulos fonéticos y gramaticales de Voces usadas en Chile. Sus críticas se concentran en la validez empírica de algunas aseveraciones particulares: por ejemplo, se muestra en desacuerdo con la afirmación de Echeverría acerca del origen mapuche del debilitamiento de /s/ implosiva, en que este sigue a Lenz. Da como prueba de lo errado de esta hipótesis que dicho fenómeno se encuentra en el sur de España así como en muchos otros lugares de América. Asimismo, señala que no es cierto que este fenómeno se dé solo en Chile ni en todos los contextos lingüísticos señalados (por ejemplo, asegura nunca haber escuchado la elisión completa antes de consonante, como en isla o escudo). Pero igualmente considera, en general, que la aproximación de Echeverría en asuntos fonéticos y gramaticales es difícilmente aceptable. Particularmente, cree que intentar hacer un inventario resulta vano porque "son tan variadas i estrafalarias las alteraciones que el pueblo ignorante comete al hablar, que es imposible clasificarlas", así como porque "las mas de ellas son frutos del capricho de cada cual" (Amunátegui Reyes 1902: 126). En cuanto al capítulo "Sintáxis", se lamenta de que "habría sido deseable que el señor Echeverría se hubiera estendido un poco mas en esta parte" (Amunátegui Reyes 1902: 128).

Su crítica del capítulo "Lexicolojía" se fundamenta en los principios que sustentan la aceptabilidad de un vocablo de formación nueva: "A mi juicio, la buena formacion de un vocablo no es suficiente credencial para que éste sea admitido sin mas trámite. Es menester todavía que el uso lo haya aceptado" (Amunátegui Reyes 1902: 129). Por otra parte, critica la "poca prolijidad" que se deja ver en este capítulo al proponer la inclusión en el Diccionario académico de muchas voces que ya estaban incorporadas (cita cerca de una cincuentena de voces) o de vocablos que dicho repertorio declara no recoger (derivados regulares y diminutivos, entre otros).

Finalmente, dedica un extenso comentario a la sección "Voces", en que se limita a "hacer algunas observaciones jenerales i a rectificar por vía de ejemplo algunos de los muchos errores que he podido notar despues de una rápida lectura" (Amunátegui Reyes 1902: 137). En primer lugar, critica el 
empeño de Echeverría por acrecentar su nomenclatura, lo cual explicaría, en su opinión, que haya incluido voces tabuizadas, que consigne numerosas lexías cuyo uso a Amunátegui Reyes no le consta (como achipilcarse, circunstanfláutico, chegre, trinca, zamarro y otros), que incluya voces de uso muy restringido (como las voces relativas al fútbol y, en general, los extranjerismos), que registre "expresiones que no son sino simples figuras de retórica" (Amunátegui Reyes 1902: 128), como aguililla 'petardista', estar frito 'haber perdido cuanto se tenía', lagarto 'astuto' y ponerse como camarón 'avergonzarse'. Asimismo, tal propósito habría motivado la inclusión de materiales propios de una enciclopedia, tales como los nombres de vinos (Huasco, Subercaseaux, Urmeneta, etc.) y los hipocorísticos (Concha, Pancho, Trini, etc.). Por otra parte, critica la lematización duplicada de ciertas variantes que podrían haberse fundido en un solo lema (desabillél deshabillé, sprit/esprit, tastabillar/trastabillar) o de acepciones que podrían haberse puesto en un solo artículo polisémico (pucho).

En segundo lugar, hace reparos a algunas voces en particular: acabóse, calentar, carái, estar frito, ¡ hupa!, ña, ño y zandunga por no ser chilenismos o americanismos (Amunátegui Reyes da ejemplos de autores españoles, además de basarse en su experiencia), balmacedista, que corresponde a la clase de los nombres efímeros de partidarios de una persona o una facción política (como aliancista), esquina en el sentido de 'lugar donde se venden comestibles', por corresponder a una interpretación errada por parte de Echeverría (además de ser usado también en España).

La valoración general del libro de Echeverría por parte de Amunátegui Reyes es negativa. Al comenzar su crítica, señala que ya otros comentaristas (Solar 1900 y Mario 1900) han conseguido "desvirtuar un poco la favorable acojida que él [Voces usadas en Chile] tuvo en el primer momento" (Amunátegui Reyes 1902: 118). Al finalizar, concluye:

En mi concepto, el señor Echeverría ha escojido un mal momento para imprimir su libro, que ha aparecido en el mismísimo tiempo en que se daba a la publicidad la última edicion del léxico académico. De aquí que en el glosario aparezcan, como neolojismos, americanismos, chilenismos, etc., multitud de voces que en el dia estan ya autorizadas. [...] Esta sola circunstancia bastaria, a mi juicio, para que se hiciera una nueva edicion de esta obra. (Amunátegui Reyes 1902: 159)

Con todo, considera loable que se publiquen obras de este tipo, por su utilidad pedagógica. Sin embargo, cree que se les debe exigir suma prolijidad, pues el error puede desorientar al lector y servir incluso de mal ejemplo. En términos generales, es esta desprolijidad lo que lo lleva a valorar en términos negativos Voces usadas en Chile. 


\section{RODOLFO LENZ, DICCIONARIO ETIMOLÓGICO (1905-1910)}

Lenz, en su Diccionario etimológico de las voces chilenas derivadas de lenguas indígenas (Lenz 1987 [1905-1910]), incluye una "Bibliografía crítica de las obras sobre americanismos". En ella reseña brevemente, entre otras obras, los principales repertorios lexicográficos publicados en Chile hasta ese momento: Rodríguez (1979 [1875]), Ortúzar (1893) y Echeverría (1900).

La crítica de Lenz, por estar inserta en una obra sobre indigenismos léxicos, se concentra en la cantidad de voces de origen indígena que cada obra registra. En opinión de Lenz, Voces usadas en Chile no representa una gran novedad al respecto: "El número de palabras indias no rejistradas con anterioridad en otros libros es insignificante, pues el autor no se ha empeñado en hacer entrar términos de historia natural, aunque tampoco los escluye rigurosamente" (Lenz 1987 [1905-1910]: 62). Asimismo, cuando habla de la utilidad de la bibliografía incluida por Echeverría, señala que "Es de sentir que el autor no haya citado sus fuentes con exactitud en las voces que llama 'americanismos'; se habria visto que las mas de las palabras indias de esta categoría no están en uso fuera del dominio de los incas" (Lenz 1987 [1905-1910]: 62).

Por otra parte, también efectúa valoraciones de otros aspectos, tales como la clasificación de las voces, la inclusión de algunos extranjerismos y la técnica definitoria empleada. Se muestra muy crítico respecto de la clasificación, señalando que

podria aceptarse si el autor los hubiera distinguido de una manera mas precisa. [...] Los términos dados no obedecen a un mismo principio de division; chilenismo i americanismo se refieren a la estensión jeográfica, neolojismo a la antigüedad, estranjerismo a la procedencia i barbarismo a la correccion lingüística o a la apreciacion literaria. [...] En jeneral, hai que decir que la clasificacion de los vocablos, así como la da Echeverría, tiene poco valor. (Lenz 1987 [1905-1910]: 62)

Critica la inclusión de algunos extranjerismos que, en su opinión, no se encuentran realmente incorporados al español de Chile, por lo cual Echeverría no debería haberlos recogido en su repertorio: "Así, por ejemplo, las numerosas voces referentes al 'foot-ball', no las conoce nadie fuera de los pocos individuos que cultivan este juego, introducido hace poco por los ingleses" (Lenz 1987 [1905-1910]: 62).

Por último critica, al igual que Fidelis del Solar, la concisión de las definiciones. Considera inadecuada, asimismo, la selección de algunos equivalentes para definir: "El criterio literario es demasiado riguroso [...]; 
piedra de esquina i piedra de moler se tildan de barbarismos i se traducen por recanton, trascanton aquélla, por metate, silleta ésta. Ninguna de estas palabras es comprensible para un chileno educado de término medio" (Lenz 1987 [1905-1910]: 63).

Con todo, su valoración global de Voces usadas en Chile es positiva. Destaca que "es un notable progreso por el arreglo mas científico i la separacion limpia entre la crítica gramatical i la lexicolójica" (Lenz 1987 [1905-1910]: 62), y que "es por ahora el mejor i mas ordenado diccionario de provincialismos que hai de pais alguno en América". (Lenz 1987 [19051910]: 63). Alaba, asimismo, la bibliografía, que considera de gran utilidad.

\section{DISCUSIÓN}

En el Cuadro 1 presentamos de manera sintética las características positivas y negativas destacadas por cada uno de los críticos de Voces usadas en Chile:

Cuadro 1. Características positivas y negativas de Echeverría (1900), según sus comentaristas

\begin{tabular}{|c|c|c|}
\hline & $\begin{array}{l}\text { Características } \\
\text { positivas }\end{array}$ & Características negativas \\
\hline Lenz y Diez (1899) & $\begin{array}{l}\text { - Prolijidad. } \\
\text { - Bibliografía. } \\
\text { - Presentación clara. }\end{array}$ & $\begin{array}{l}\text { - Podría haberse dado arreglo } \\
\text { más científico a las secciones } \\
\text { de morfología y sintaxis. }\end{array}$ \\
\hline Solar (1900) & $\begin{array}{l}\text { - Valor pedagógico. } \\
\text { - Cantidad de voces. } \\
\text { - Diversidad de voces }\end{array}$ & $\begin{array}{l}\text { - Definiciones muy breves, por } \\
\text { tanto poco claras. } \\
\text { - Falta entrega de equivalentes en } \\
\text { extranjerismos. } \\
\text { - Abreviaturas a veces ambiguas } \\
\text { y poco claras } \\
\text { - Tratamiento de ítems léxicos } \\
\text { particulares (chilenismos } \\
\text { falsos, supresiones, adiciones y } \\
\text { rectificaciones). } \\
\text { - Inclusión de voces tabuizadas. }\end{array}$ \\
\hline
\end{tabular}




\begin{tabular}{|c|c|c|}
\hline Mario (1900) & - Cantidad de voces. & $\begin{array}{l}\text { - Inclusión innecesaria de algunos } \\
\text { extranjerismos que no tienen } \\
\text { real arraigo en el uso chileno. } \\
\text { - Tratamiento de ítems léxicos } \\
\text { particulares. } \\
\text { - Inclusión de voces tabuizadas. } \\
\text { - Poca prolijidad en el estable- } \\
\text { cimiento de contrastes con el } \\
D R A E \text {. } \\
\text { - Poca prolijidad, en general. }\end{array}$ \\
\hline Anónimo (1900) & $\begin{array}{l}\text { - Actualizado. } \\
\text { - Muestra influen- } \\
\text { cia extranjera e in- } \\
\text { doamericana en el } \\
\text { español de Chile. } \\
\text { - Información fónica } \\
\text { y gramatical. } \\
\text { - Bibliografía. }\end{array}$ & \\
\hline $\begin{array}{l}\text { Amunátegui Reyes } \\
\text { (1902) }\end{array}$ & - Valor pedagógico. & $\begin{array}{l}\text { - Inclusión de voces tabuizadas. } \\
\text { - Algunas afirmaciones del autor } \\
\text { son empíricamente infundadas. } \\
\text { - No es realista intentar hacer } \\
\text { una clasificación de fenómenos } \\
\text { fónicos y gramaticales. } \\
\text { - El capítulo de sintaxis es muy } \\
\text { corto. } \\
\text { - El capítulo de lexicología se basa } \\
\text { en criterios de aceptabilidad } \\
\text { insuficientes. } \\
\text { - Excesivo empeño por acrecentar } \\
\text { nomenclatura. } \\
\text { - Lematizaciones duplicadas. } \\
\text { - Separación en distintos artículos } \\
\text { de acepciones que podrían haber } \\
\text { ido en uno solo. } \\
\text { - Poca prolijidad en el estable- } \\
\text { cimiento de contrastes con el } \\
D R A E \text {. }\end{array}$ \\
\hline
\end{tabular}




\begin{tabular}{|c|c|c|}
\hline Lenz (1905) & $\begin{array}{l}\text { - Bibliografía. } \\
\text { - Arreglo más científi- } \\
\text { co que otros diccio- } \\
\text { narios. } \\
\text { - Separación metodo- } \\
\text { lógica entre gramá- } \\
\text { tica y lexicología. }\end{array}$ & $\begin{array}{l}\text { - Atribuye carácter de americanis- } \\
\text { mos a palabras de uso geográfi- } \\
\text { co muy restringido. } \\
\text { - Clasificación de las voces. } \\
\text { - Inclusión innecesaria de algunos } \\
\text { extranjerismos que no tienen } \\
\text { real arraigo en el uso chileno. } \\
\text { - Definiciones muy breves, por } \\
\text { tanto poco claras. } \\
\text { - Algunos de los equivalentes con } \\
\text { que define fueron seleccionados } \\
\text { con poco acierto. }\end{array}$ \\
\hline
\end{tabular}

Como es claro, en la valoración de Voces usadas en Chile por parte de sus contemporáneos sobresalen numéricamente las características consideradas negativas por sobre las positivas. Solo el reseñista anónimo de The Nation encuentra nada más que cualidades positivas en esta obra.

Entre sus características positivas, resulta especialmente notable para los comentaristas la inclusión de una bibliografía sobre el español y sus variedades. Esta sección, de 21 páginas, contiene aproximadamente 150 referencias agrupadas por criterios geográficos ("América del Norte", "América Central", "América del Sur", y luego por países dentro de cada una de ellas) y temáticos (contiene una sección en que agrupa las obras sobre "Corrección del Lenguaje"). Además de dar cuenta de un laborioso trabajo de revisión de obras previas, este rasgo probablemente daba a entender a los lectores que el contenido de Voces usadas en Chile tenía un sólido respaldo empírico. Echeverría había publicado esta bibliografía en 1897, como un pequeño volumen independiente, bajo el título Sobre lenguaje, y al momento de publicarla como parte de Voces usadas en Chile la actualizó, como revela la inclusión de algunas obras posteriores a 1897. Las cartas de Echeverría a Rodolfo Lenz revelan que probablemente solo a partir de esta misma fecha comenzó a emplear efectivamente su biblioteca para cotejar el material que tenía recogido con el uso del resto de América (Rojas 2011: 356).

Otro rasgo que valoran positivamente al menos un par de los comentaristas es la cantidad de voces incluidas, 4061 entradas (Becerra, Castro y Garrido 2007: 139). En comparación con Rodríguez (1979 [1875]), que contiene 1100 monografías (Becerra, Castro y Garrido 2007: 51), efectivamente, el aumento del caudal léxico es considerable; sin embargo, no supera cuantitativamente a Ortúzar (1893), que contiene 5244 entradas (Becerra, Castro y Garrido 2007: 101). El fetichismo de la cantidad, como señala Béjoint (2000: 114), es hoy 
en día característico de la percepción no especializada acerca de las obras lexicográficas: mientras más palabras o expresiones tenga un diccionario, mejor les parece, en principio, a los usuarios. No es de extrañar, entonces, que esta idea aparezca también entre algunos de los comentaristas de Echeverría. Cabe destacar, sin embargo, que otros comentaristas subrayan la contraparte negativa de la gran cantidad de voces incluidas. Solar (1900), en particular, atribuye a esta característica la brevedad de las definiciones, que terminan siendo, en su opinión, poco informativas.

Entre las características negativas señaladas, sobresale la inclusión de voces tabuizadas. Lenz (1987 [1905-1910]) es el único que no manifiesta opinión alguna al respecto: años más tarde el filólogo alemán expresará que el diccionario general de la lengua española, que, según su opinión, debe recoger todo lo que se dice, debería también incluir "las palabras que se excluyen de la conversación de la gente culta, por considerarse indecentes" (Lenz 1926: 40). La visión descriptivista de Lenz (en el sentido de que no considera valoraciones al momento de seleccionar elementos para conformar la nomenclatura) puede haber influido en la postura de Echeverría frente a las voces tabuizadas. El autor de Voces usadas en Chile se justifica de la siguiente manera:

En nuestra obra figuran diversos vocablos o locuciones que algunos pudieran tachar de indecorosos u obscenos. Les hemos dado cabida por dos razones: desde luego, por juzgar que todo trabajo literario, cualquiera que sea su objeto, no envuelve intrinsicamente idea alguna nociva o vituperable, siempre que se concrete a enseñar la verdad; i en segundo término, porque dar a conocer en detalle las diversas voces proferidas constantemente en una determinada rejion, exije fidelidad completa de esposicion, esto es, no omitir ninguna y precisar su significado; a no ser ello exacto, todos los Léxicos merecerian, en lo que a decencia de lenguaje respecta, la fea nota de inmoralidad o de ocasionados a pervertir costumbres. Fijar el valor propio de dicciones que incluyen desdorosos conceptos, no se encamina a sujerir ideas contrarias a la nobleza de espresion, ni mucho menos recomendar el empleo de aquellas: labor semejante es sólo el reconocimiento de un hecho. Ningun saber humano es inmoral: llega a serlo cuando su aplicacion es ilejitima. (Echeverría 1900: xxi-xxii)

Mario (1900), Solar (1900) y Amunátegui Reyes (1902), como es claro, no comparten esta opinión. En estos comentaristas podemos ver reflejada una idea que ha pervivido hasta la actualidad en la visión popular acerca del diccionario: que este debe actuar como "guardián de los valores morales e ideológicos de la sociedad" (Béjoint 2000: 124; traducción nuestra). 
Este autor señala que la tradición de excluir por razones morales las voces referidas al sexo y las funciones excretivas fue iniciada por la Academia Francesa en su Dictionnaire de 1694; antes de esto, los diccionarios ingleses, por ejemplo, no tenían reparo en incluirlas. Esta tendencia se ha revertido, tanto en la tradición francesa como en la inglesa, solo desde la segunda mitad del siglo XX, y de manera paulatina. En la tradición hispánica, la influencia francesa se dejó sentir desde muy temprano: ya el Diccionario de autoridades (1726-1739) señala que "se escusarán todas las palabras que significan desnudadamente objeto indecente", e incluso en el Tesoro de la lengua castellana o española de Covarrubias, bastante anterior (1611), puede apreciarse una cierta pudibundez (Ruhstaller 1997).

Otra característica percibida negativamente por varios comentaristas es que Echeverría incluyó voces que carecían de suficiente arraigo en el uso nacional como para haber sido consideradas "voces usadas en Chile": las expresiones cuestionadas, por el contrario, sobresalen por ser más bien voces no usadas en Chile. En especial, muchos de los extranjerismos son objeto de esta crítica, de manera que los comentarios positivos del reseñista anónimo de The Nation pierden algo de sustento.

Sin embargo, en el Cuadro 1 no queda reflejada de manera suficiente la cualidad negativa que durante mayor cantidad de páginas ocupa a los comentaristas analizados: el tratamiento normativo dado a ítems léxicos particulares. Es muy representativo el caso de Mario (1900), quien comenta detalladamente 214 voces respecto de cuya descripción o tratamiento normativo disiente. Las ideas con que Mario sustenta sus disentimientos son de diversos órdenes: por ejemplo, acude a argumentos de autoridad para justificar que una palabra es propia de la lengua ejemplar: así, si una palabra como canon está recogida en el Diccionario de la Real Academia Española, no merece, en su opinión, la calificación negativa ("barbarismo") que le da Echeverría. En otros casos, Mario intenta refutar a Echeverría contraponiendo lo que este dice al uso "real", representado por su propio conocimiento o por la autoridad descriptiva de personalidades como Camilo Ortúzar o Claudio Gay. En otras ocasiones simplemente recurre al razonamiento sobre los mecanismos internos de la lengua española (el "genio del idioma"), que contienen ciertas potencialidades legítimas realizadas en las palabras discutidas.

En nuestra opinión, los textos críticos de la obra de Echeverría pueden considerarse como vehículos de la negociación de normas que tenía lugar en el Chile de fines del siglo XIX, dentro del proceso de estandarización lingüística que estaba ocurriendo en la época. En estudios recientes sobre la constitución de lenguas estándares y de normas (Andersen 2009) se ha puesto de relieve que la selección de rasgos lingüísticos para la conformación 
y actualización de una variedad ejemplar ocurre no únicamente mediante la imposición autoritaria y unidireccional por parte de agentes premunidos de poder, sino también mediante la negociación, implícita o explícita, entre los miembros de una comunidad idiomática. Una comunidad lingüística, desde cierto punto de vista, puede concebirse como un sistema multiagente normativo (Boella y Torre 2007), pues permite a los miembros de una comunidad coordinar sus acciones mediante obligaciones y normas, a la vez que permite la creación de nuevas normas; es esta última posibilidad lo que hace necesario establecer mecanismos de negociación para determinar qué propuestas son finalmente acogidas en el seno de la sociedad.

Al participar en la negociación de normas lingüísticas, los hablantes plantean sus propias propuestas o evalúan propuestas efectuadas por otros hablantes respecto de la adecuación o legitimidad social de un rasgo lingüístico en particular, entablando una actividad comunicativa dialógica en la cual el discurso argumentativo adquiere un marcado protagonismo. Aunque Andersen (2009) ha puesto énfasis en la negociación implícita, los textos que analizamos corresponden a negociaciones de tipo explícito, es decir, manifiestas abiertamente como tales:

La negociación puede entenderse como una manera de promover las hipótesis personales respecto del carácter de las normas en desmedro de otras hipótesis existentes en la comunidad lingüística. Nos encontramos frente a este caso cuando las normas lingüísticas se transforman en objeto de debate público en columnas de periódicos, programas radiales o blogs de Internet. [...] Estos debates pueden denominarse negociaciones de normas explícitas (las normas en cuestión son traídas a colación específicamente para su escrutinio) y constituyen una característica típica de las sociedades de lenguaje modernas. (Lunde y Paulsen 2009: 9; traducción nuestra)

El concepto de negociación de normas, específicamente la negociación de tipo explícito, constituye, en nuestra opinión, una importante herramienta teórica para comprender a cabalidad el discurso metalingüístico contenido en los diccionarios de provincialismos americanos del siglo XIX. Gran parte de este discurso es argumentativo: contiene una discusión explícita de argumentos planteados por otros autores, presentando argumentos para refutarlos o corroborarlos, o bien planteando argumentos para defender nuevas propuestas. Esto puede apreciarse nítidamente en Rodríguez (1979 [1875]), mas no así en Echeverría (1900), pues este último presenta un grado mucho mayor de condensación textual lexicográfica (Bustos Plaza y Wiegand 2005-2006). Los comentaristas de Echeverría (1900), en cambio, muestran una configuración textual más parecida, en este sentido, al estilo de 
Rodríguez (1979 [1875]), en virtud de lo cual presentan de manera explícita sus justificaciones.

La fuerte presencia de actos de negociación de normas en los comentaristas de Voces usadas en Chile tiene sentido si se considera el periodo lingüísticohistórico en que se enmarcan. Según Matus, Dargham y Samaniego (1992), la comunidad hispanohablante chilena, a fines del s. XIX, ya había entrado en un proceso de estandarización circunstanciada, caracterizado por hitos como la publicación de obras que se proponían codificar la lengua española (como la Gramática de Andrés Bello, de 1847) y la oficialización de la lengua española en la enseñanza chilena, entre otros. Este proceso, por estar aún no acabado en la fecha de publicación de la obra de Echeverría, se refleja abundantemente en las obras que la comentan. El subgénero de los "comentarios" de repertorios lexicográficos, de este modo, se constituía como un espacio discursivo idóneo para discutir una norma lingüística emergente de carácter local (el español se ha configurado como una lengua de norma pluricéntrica, es decir, distribuida entre varios centros; cf. Lebsanft 2007 y Thompson 1992), en complementariedad, claro, con los diccionarios y obras lexicográficas mayores del contexto chileno, dentro de los cuales también tenía lugar esta discusión. En particular, la negociación de normas que se despliega en estos textos concierne principalmente al proceso de selección de rasgos lingüísticos (Ammon 2004; Trudgill y Hernández 2007, s. v. selección), pues lo que estaba sujeto a discusión era cuáles usos lingüísticos chilenos eran aptos para formar parte de una variedad estándar local.

Puesto que nuestro objetivo en este artículo era describir la recepción de la obra de Echeverría y las ideas que la sustentaron, no nos podemos hacer cargo al mismo tiempo del estudio detallado de la negociación de normas lingüísticas manifestada en el tejido intertextual desplegado por Echeverría y sus comentaristas. Nos limitamos, por tanto, a apuntar la idea de que la crítica metalexicográfica chilena del s. XIX se relaciona estrechamente con este tipo de negociación.

\section{CONCLUSIONES}

Los comentarios a Voces usadas en Chile dan cuenta de que los lectores se enfrentaron a la obra de Echeverría de manera congruente con el periodo de la lexicografía chilena y el clima de opinión lingüística en que se enmarcan: juzgaron este libro principalmente de acuerdo con su valor como obra normativa. Las inadecuaciones descriptivas que varios de los comentaristas 
señalan en Voces usadas en Chile no parecen haber tenido relevancia per se (como observaciones relativas únicamente a su valor descriptivo), sino porque implicaban una merma de su valor normativo. Por otra parte, las aseveraciones aparentemente descriptivas de Echeverría eran interpretadas por los lectores como indicaciones sobre cuáles eran o podían ser las formas propias de la lengua ejemplar de la época, de modo que, si no estaban de acuerdo con estas indicaciones, podían entablar una disputa argumentativa, es decir, plantear un proceso de negociación de normas lingüísticas, de acuerdo con lo esperable en una situación de conformación de una variedad estándar local. Por esta misma razón, las ideas acerca de la lengua que se dejan ver en estos comentarios son principalmente de índole normativa: por ejemplo, al considerar que las palabras tabuizadas no deben ser incluidas en el diccionario, se deja ver una idea de tipo prescriptivo respecto de lo que debe ser la lengua.

En nuestra opinión, al menos desde el punto de vista de cómo los lectores interactuaban con el texto, el descriptivismo de la obra de Echeverría era más aparente que efectivo. Por encontrarse Voces usadas en Chile inserta en una tradición de obras que tenían propósito normativo, sus lectores no podían sino hacer una lectura sobre el trasfondo de dicha tradición. Si es que la intención de Echeverría era desligarse de la tradición normativa chilena del s. XIX (intención aún no suficientemente esclarecida, en nuestra opinión, aunque dada como un hecho ${ }^{4}$, por ejemplo, por Chávez 2010: 62), no tuvo suficientemente en cuenta el horizonte de expectativas de sus potenciales lectores. Esto puede explicar la reacción predominantemente negativa que suscitó Voces usadas en Chile entre sus contemporáneos.

4 Sabemos, por una carta que Echeverría le envió a Lenz en 1894 (Rojas 2011: 353354), que el abogado concibió originalmente su "vocabulario" como una sección de una obra mayor y colectiva que incluiría también una adaptación chilena del Diccionario ortográfico del español José Manuel Marroquín y escritos sobre ortografía de Lenz. Esto apunta a que el propósito pedagógico y normativo común a las obras de la época estuvo también presente en Voces usadas en Chile desde su concepción. Por otra parte, diversos pasajes del "Prólogo", como el que citamos a continuación, dan cuenta de una intención similar a la de Rodríguez (1979 [1875]): “. . . nos es satisfactorio ofrecer a los aficionados al estudio de nuestro idioma algunas modestas apuntaciones sobre los vicios que se deslizan en nuestro lenguaje corriente" (Echeverría 1900: xiv). 


\section{REFERENCIAS BIBLIOGRÁFICAS}

Alfero, Daniela, José J. Atria y Enrigue Sologuren. 2008. Lexicografía chilena finisecular. Voces usadas en Chile de Aníbal Echeverría y Reyes (1900). Onomázein 18(2): 113-137.

AMmON, UlRICH. 2004. Standard variety / Standardvarietät. En Ulrich Ammon, Norbert Dittmar, Klaus J. Mattheier y Peter Trudgill (eds.). Sociolinguistics: An International Handbook of the Science of Language and Society / Soziolinguistik: Ein internationales Handbuch zur Wissenschaft von Sprache und Gesellschaft, $2^{\mathrm{a}}$ edición completamente revisada y extendida, vol. 1, pp. 273-283. Berlín/Nueva York: Walter de Gruyter.

Amunátegui Reyes, Miguel Luis. 1902. Voces usadas en Chile por D. Aníbal Echeverría i Reyes. En Críticas y charlas, pp. 115-160. Santiago de Chile: Imprenta Cervantes.

-1924-1927. Observaciones y enmiendas a un Diccionario, aplicables también a otros. Tomo I: 1924; tomo II: 1925, tomo III: 1927. Santiago de Chile: Imprenta Universitaria.

Andersen, Henning. 2009. Living norms. En Ingunn Lunde y Martin Paulsen (eds.). Poets to Padonki: Linguistic Authority \& Norm Negotiation in Modern Russian Culture, pp. 18-33. Slavica Bergensia 9, Bergen.

Anónimo. 1900. Voces usadas en Chile, por Aníbal Echeverría i Reyes. The Nation, vol. 70, n. 1809: 173.

Azorín Fernández, Dolores. 2003. La lexicografía como disciplina lingüística. En Antonia M. Medina Guerra (coord.). Lexicografia española, pp. 31-52. Barcelona: Ariel.

Becerra, Yanira, Verena Castro y Claudio Garrido, 2007. Tres repertorios léxicos diferenciales del español de Chile en el s. XIX. Un estudio metalexicográfico. Tesis de licenciatura, Universidad de Chile.

Bello, ANDRÉs. 1847. Gramática de la lengua castellana destinada al uso de los americanos. Santiago de Chile.

BÉJoInt, Henri. 2000. Modern Lexicography: An Introduction. Oxford: Oxford University Press.

Blommaert, Jan (ed.). 1999. Language Ideological Debates. Berlín/Nueva York: Mouton de Gruyter.

Boella, Guido y LeEndert W.N. van Der Torre. 2007. Norm negotiation in multiagent systems. International Journal of Cooperative Information Systems 16(2): 97-122.

Bustos Plaza, Alberto y Herbert Ernst Wiegand. 2005-2006. Condensación textual lexicográfica: esbozo de una concepción integral. Revista de Lexicografía XII: 7-46.

Chávez, Soledad. 2009. Diccionarios del español de Chile en su fase precientífica: un estudio metalexicográfico. Tesis de maestría, Universidad de Chile.

2010. Ideas lingüísticas en prólogos de diccionarios diferenciales del español de Chile. Etapa 1875-1928. Boletín de Filología de la Universidad de Chile XLV(2): 49-69.

Coupland, Nikolas y AdAm JaWORSKi. 2004. Sociolinguistic perspectives on metalanguage: Reflexivity, evaluation and ideology. En Nikolas Coupland, Adam Jaworski y Dariusz Galasinski (eds.). Metalanguage: Social and Ideological Perspectives, pp. 15-51. Berlín: Mouton de Gruyter.

Davies, Winifred y Nils Langer. 2006. The Making of Bad Language. Frankfurt: Peter Lang. Echeverría, Aníbal. 1900. Voces usadas en Chile. Santiago de Chile: Imprenta Elzeviriana. Hausmann, Franz J. 1989. Pour une histoire de la métalexicographie. En Franz J. Hausmann, Oskar Reichmann, Herbert E. Wiegand y Ladislav Zgusta (eds.). Wörterbücher / Dictionaries / Dictionnaires. Ein internationales Handbuch zur Lexikographie / An International Encyclopedia of Lexicography / Enciclopédie internationale de lexicographie, tomo 1, pp. 216-224. Berlín/Nueva York: Mouton de Gruyter. 
Kroskrity, PaUl V. 2010. Language ideologies - Evolving perspectives. En Jürgen Jaspers, Jan-Ola Östman y Jef Verschueren (eds.). Society and Language Use, pp. 192-211. Ámsterdam/Filadelfia: John Benjamins.

Lara, Luis Fernando. 1997. Teoría del diccionario monolingüe. México: El Colegio de México.

2003. El diccionario y sus disciplinas. Revista Internacional de Lingüistica Iberoamericana 1(1): 35-49.

Lebsanft, Franz. 2007. Norma pluricéntrica del español y Academias de la Lengua. En Christopher F. Laferl y Bernhard Pöll (eds.). Amerika und die Norm. Literatursprache als Modell?, pp. 227-246. Tübingen: Max Niemeyer Verlag.

Lenz, Rodolfo. 1987 [1905-1910]. Diccionario etimológico de las voces chilenas derivadas de lenguas indígenas americanas. Edición de Mario Ferreccio. Santiago: Universidad de Chile.

1926. Problemas del diccionario castellano en América. Separata del Boletín del Instituto de Filología, I/3-4. Buenos Aires: Imprenta de la Universidad de Buenos Aires.

Lunde, Ingunn y Martin Paulsen. 2009. Introduction. En Ingunn Lunde y Martin Paulsen (eds.). Poets to Padonki: Linguistic Authority \& Norm Negotiation in Modern Russian Culture, pp. 7-17. Slavica Bergensia 9.

Mario [José Ramón SAAVEdRa]. 1900. Reparos a Voces usadas en Chile por Aníbal Echeverría y Reyes. El Porvenir (Santiago), 8122-8129, 8131-8134, 8139.

Matus, Alfredo. 1994. Períodos de la lexicografía diferencial del español de Chile. En Actas del X Congreso de la Asociación de Academias de la Lengua Española, pp. 189-199. Madrid: Real Academia Española/Espasa Calpe.

Matus, Alfredo, Soledad Dargham y José Luis Samaniego. 1992. Notas para una historia del español en Chile. En César Hernández A. (coord.). Historia y presente del español de América, pp. 543-564. Valladolid: Junta de Castilla y León, PABECAL.

Medina, José Toribio. 1928. Chilenismos. Apuntes lexicográficos. Santiago de Chile: Imp. Universo.

Ortúzar, Camilo. 1893. Diccionario manual de locuciones viciosas y de correcciones del lenguaje con indicación del valor de algunas palabras y ciertas nociones gramaticales. San Benigno Canavese: Imprenta Salesiana.

Rodríguez, Zorobabel. 1979 [1875]. Diccionario de chilenismos. Edición facsimilar. Valparaíso: Editorial Universitaria de Valparaíso.

RoJAS, DARío. 2010. Estandarización lingüística y pragmática del diccionario: forma y función de los "diccionarios de provincialismos" chilenos. Boletín de Filología de la Universidad de Chile XLV(1): 209-233.

2011. Voces usadas en Chile (1900): las cartas de Aníbal Echeverría y Reyes a Rodolfo Lenz. Onomázein 24(2): 349-361.

Rojas Carrasco, Guillermo. 1940. Filología chilena. Guía bibliográfica y crítica. Santiago de Chile: Universo.

Ruhstaller, Stefan. 1997. El tratamiento lexicográfico de las voces referentes a la sexualidad en el Diccionario de Autoridades y en el Tesoro de Covarrubias. En Luis Gómez Canseco et al. (eds.). El sexo en la literatura, pp. 143-150. Huelva: Universidad de Huelva.

Silva Castro, Raúl. 1951. Miguel Luis Amunátegui Reyes. 1862-1949. Santiago de Chile: Editorial Jurídica de Chile.

Solar, Fidelis Del. 1876. Reparos al "Diccionario de chilenismos" del señor don Zorobabel Rodríguez. Santiago de Chile: Imprenta de Federico Schrebler.

1900. Voces usadas en Chile. Juicio crítico de la obra que con este título acaba de dar a luz don Aníbal Echeverría y Reyes. Santiago de Chile: Imprenta Moderna. 
Thompson, R. W. 1992. Spanish as a pluricentric language. En Michael Clyne (ed.). Pluricentric Languages: Differing Norms in Different Nations, pp. 45-70. Berlín/Nueva York: Mouton de Gruyter.

Trudgill, Peter y y Juan Manuel Hernández. 2007. Diccionario de sociolingüística. Madrid: Gredos.

Wiegand, Herbert Ernst. 1984. On the Structure and Contents of a General Theory of Lexicography. En R. R. K. Hartmann (ed.). LEXeter Proceedings. Papers from the International Conference on Lexicography at Exeter 9-12 September 1983, pp. 13-30. Tübingen: Max Niemeyer Verlag.

Wilton, Antje y Holger Wochele. 2011. Linking past and present: A view of historical comments about language. En Antje Wilton y Martin Stegu (eds.). Applied Folk Linguistics, pp. 55-67. AILA Review 24. 